British Journal of Mathematics \& Computer Science 19(3): 1-10, 2016, Article no.BJMCS.29914 ISSN : 2231-0851

SCIENCEDOMAIN international www.sciencedomain.org

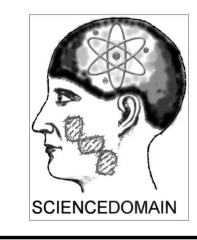

\title{
Existence and Nonexistence of Positive Solutions for a System of Higher-Order Differential Equations with Integral Boundary Conditions
}

\author{
Rodica Luca ${ }^{*}$ and Alexandru Tudorache ${ }^{2}$ \\ ${ }^{1}$ Department of Mathematics, Gh. Asachi Technical University, Iasi 700506, Romania. \\ ${ }^{2}$ Faculty of Computer Engineering and Automatic Control, Gh. Asachi Technical University, \\ Iasi 700050, Romania. \\ Authors' contributions
}

This work was carried out in collaboration between both authors. Both authors read and approved the final manuscript.

Article Information

DOI: $10.9734 / B J M C S / 2016 / 29914$ Editor(s):

(1) Zuomao Yan, Department of Mathematics, Hexi University, China. Reviewers:

(1) Rashmi Awad, Devi Ahilya University, Indore, India. (2) Peiluan Li, Henan University of Science and Technology, China. Complete Peer review History: http://www.sciencedomain.org/review-history/16681

\section{Abstract \\ In this paper, we investigate the existence and nonexistence of positive solutions for a system of nonlinear higher-order ordinary differential equations with Riemann-Stieltjes integral boundary conditions which contain some positive constants.}

Keywords: Higher-order differential equations; integral boundary conditions; positive solutions; existence; nonexistence.

2010 Mathematics Subject Classification: 34B10, 34B18.

\footnotetext{
${ }^{*}$ Corresponding author: E-mail: rlucatudor@yahoo.com;
} 


\section{Introduction}

We consider the system of nonlinear higher-order ordinary differential equations

$$
\left\{\begin{array}{l}
u^{(n)}(t)+a(t) f(v(t))=0, \quad t \in(0, T), \\
v^{(m)}(t)+b(t) g(u(t))=0, \quad t \in(0, T),
\end{array}\right.
$$

with the integral boundary conditions

$$
\left\{\begin{array}{l}
u(0)=\int_{0}^{T} u(s) d H_{1}(s)+a_{0}, u^{\prime}(0)=\cdots=u^{(n-2)}(0)=0, \quad u(T)=\int_{0}^{T} u(s) d H_{2}(s), \\
v(0)=\int_{0}^{T} v(s) d K_{1}(s)+b_{0}, \quad v^{\prime}(0)=\cdots=v^{(m-2)}(0)=0, \quad v(T)=\int_{0}^{T} v(s) d K_{2}(s),
\end{array}\right.
$$

where $T>0, n, m \in \mathbb{N}, n, m \geq 2$, the integrals from the boundary conditions $(B C)$ are RiemannStieltjes integrals, $H_{i}, K_{i}:[0, T] \rightarrow \mathbb{R}, i=1,2$ are functions of bounded variation, and $a_{0}$ and $b_{0}$ are positive constants. In the case $n=2$ or $m=2$ the boundary conditions above are of the form $u(0)=\int_{0}^{T} u(s) d H_{1}(s)+a_{0}, u(T)=\int_{0}^{T} u(s) d H_{2}(s)$, or $v(0)=\int_{0}^{T} v(s) d K_{1}(s)+b_{0}$, $v(T)=\int_{0}^{T} v(s) d K_{2}(s)$, respectively, that is, without conditions on the derivatives of $u$ and $v$ at point 0 . The Riemann-Stieltjes integral boundary conditions $(B C)$ cover the Riemann integral boundary conditions (when the functions $H_{1}, H_{2}, K_{1}, K_{2}$ are continuously differentiable functions), the multi-point boundary conditions (when the functions $H_{1}, H_{2}, K_{1}, K_{2}$ are step functions), and combinations between them.

By using the Schauder fixed point theorem and some properties of the associated Green's functions, we prove the existence of positive solutions of problem $(S)-(B C)$ for $a_{0}, b_{0}$ sufficiently small. By a positive solution of $(S)-(B C)$ we mean a pair of functions $(u, v) \in C^{n}\left([0, T] ; \mathbb{R}_{+}\right) \times C^{m}\left([0, T] ; \mathbb{R}_{+}\right)$ satisfying $(S)$ and $(B C)$ with $u(t)>0, v(t)>0$ for all $t \in[0, T)$. Then we give sufficient conditions for the nonexistence of positive solutions for this problem. Similar results for other three boundary value problems are also presented. System $(S)$ with the multi-point boundary conditions $u(0)=u^{\prime}(0)=\cdots=u^{(n-2)}(0)=0, u(T)=\sum_{i=1}^{p-2} a_{i} u\left(\xi_{i}\right)+a_{0}, v(0)=v^{\prime}(0)=\cdots=v^{(m-2)}(0)=0$, $v(T)=\sum_{i=1}^{q-2} b_{i} v\left(\eta_{i}\right)+b_{0},\left(a_{0}, b_{0}>0\right)$ has been investigated in [1].

Boundary value problems with positive solutions describe many phenomena in the applied sciences such as the nonlinear diffusion generated by nonlinear sources, thermal ignition of gases and concentration in chemical or biological problems. Problems with integral boundary conditions arise in thermal conduction problems, semiconductor problems and hydrodynamic problems. In the last decades, many authors investigated differential equations or systems of differential equations with integral boundary conditions, for which they prove the existence, multiplicity and nonexistence of positive solutions by using various methods, such as fixed point theorems in cones, the LeraySchauder continuation theorem, nonlinear alternatives of Leray-Schauder type, fixed point index theory and coincidence degree theory (see, for example, [2], [3], [4], [5], [6], [7], [8], [9], [10], [11], [12], [13], [14], [15]).

The paper is organized as follows. Section 2 contains some auxiliary results. The main theorems are presented in Section 3, and in Section 4 we give an example which supports our results.

\section{Auxiliary Results}

In this section we present some auxiliary results from [16] related to the following $n$-order differential equation

$$
u^{(n)}(t)+z(t)=0, \quad t \in(0, T)
$$


with the integral boundary conditions

$$
u(0)=\int_{0}^{T} u(s) d H_{1}(s), u^{\prime}(0)=\cdots=u^{(n-2)}(0)=0, u(T)=\int_{0}^{T} u(s) d H_{2}(s),
$$

where $n \in \mathbb{N}, n \geq 2$, and $H_{1}, H_{2}:[0, T] \rightarrow \mathbb{R}$ are functions of bounded variation. If $n=2$, the condition (2.2) has the form $u(0)=\int_{0}^{T} u(s) d H_{1}(s), u(T)=\int_{0}^{T} u(s) d H_{2}(s)$.

Lemma 2.1. ([16]) If $H_{1}, H_{2}$ are functions of bounded variation, $\Delta_{1}=\left(1-\int_{0}^{T} d H_{2}(s)\right) \times$ $\times \int_{0}^{T} s^{n-1} d H_{1}(s)+\left(1-\int_{0}^{T} d H_{1}(s)\right)\left(T^{n-1}-\int_{0}^{T} s^{n-1} d H_{2}(s)\right) \neq 0$, and $z \in C[0, T]$, then the solution $u \in C^{n}[0, T]$ of (2.1)-(2.2) is given by $u(t)=\int_{0}^{T} G_{1}(t, s) z(s) d s$, where the Green's function $G_{1}$ is defined by

$$
\begin{aligned}
& G_{1}(t, s)=g_{1}(t, s)+\frac{1}{\Delta_{1}}\left[\left(T^{n-1}-t^{n-1}\right)\left(1-\int_{0}^{T} d H_{2}(\tau)\right)\right. \\
& \left.+\int_{0}^{T}\left(T^{n-1}-\tau^{n-1}\right) d H_{2}(\tau)\right] \int_{0}^{T} g_{1}(\tau, s) d H_{1}(\tau) \\
& +\frac{1}{\Delta_{1}}\left[t^{n-1}\left(1-\int_{0}^{T} d H_{1}(\tau)\right)+\int_{0}^{T} \tau^{n-1} d H_{1}(\tau)\right] \int_{0}^{T} g_{1}(\tau, s) d H_{2}(\tau),
\end{aligned}
$$

for all $(t, s) \in[0, T] \times[0, T]$, and

$$
g_{1}(t, s)=\frac{1}{(n-1) ! T^{n-1}}\left\{\begin{array}{l}
t^{n-1}(T-s)^{n-1}-T^{n-1}(t-s)^{n-1}, \quad 0 \leq s \leq t \leq T, \\
t^{n-1}(T-s)^{n-1}, \quad 0 \leq t \leq s \leq T .
\end{array}\right.
$$

Lemma 2.2. ([16]) The function $g_{1}$ given by (2.4) has the properties:

a) $g_{1}:[0, T] \times[0, T] \rightarrow \mathbb{R}$ is a continuous function, $g_{1}(t, s) \geq 0$ for all $(t, s) \in[0, T] \times[0, T]$, $g_{1}(t, s)>0$ for all $(t, s) \in(0, T) \times(0, T)$.

b) $g_{1}(t, s) \leq h_{1}(s)$ for all $(t, s) \in[0, T] \times[0, T]$, where $h_{1}(s)=\frac{s(T-s)^{n-1}}{(n-2) ! T}$.

c) $g_{1}(t, s) \geq k_{1}(t) h_{1}(s)$ for all $(t, s) \in[0, T] \times[0, T]$, where

$$
k_{1}(t)=\min \left\{\frac{(T-t) t^{n-2}}{(n-1) T^{n-1}}, \frac{t^{n-1}}{(n-1) T^{n-1}}\right\}= \begin{cases}\frac{t^{n-1}}{(n-1) T^{n-1}}, & 0 \leq t \leq T / 2, \\ \frac{(T-t) t^{n-2}}{(n-1) T^{n-1}}, & T / 2 \leq t \leq T .\end{cases}
$$

Lemma 2.3. ([16]) Assume that $H_{1}, H_{2}:[0, T] \rightarrow \mathbb{R}$ are nondecreasing functions, $H_{1}(T)-H_{1}(0)<$ 1 and $H_{2}(T)-H_{2}(0)<1$. Then the Green's function $G_{1}$ of problem (2.1)-(2.2), given by (2.3), satisfies the properties

a) $G_{1}:[0, T] \times[0, T] \rightarrow \mathbb{R}$ is a continuous function, $G_{1}(t, s) \geq 0$ for all $(t, s) \in[0, T] \times[0, T]$, and $G_{1}(t, s)>0$ for all $(t, s) \in(0, T) \times(0, T)$.

b) $G_{1}(t, s) \leq J_{1}(s), \forall(t, s) \in[0, T] \times[0, T]$, where $J_{1}(s)=\tau_{1} h_{1}(s), s \in[0, T]$ and

$$
\begin{aligned}
& \tau_{1}=1+\frac{1}{\Delta_{1}}\left[T^{n-1}\left(1-H_{2}(T)+H_{2}(0)\right)+\int_{0}^{T}\left(T^{n-1}-\tau^{n-1}\right) d H_{2}(\tau)\right] \\
& \times\left(H_{1}(T)-H_{1}(0)\right)+\frac{1}{\Delta_{1}}\left[T^{n-1}\left(1-H_{1}(T)+H_{1}(0)\right)+\int_{0}^{T} \tau^{n-1} d H_{1}(\tau)\right] \\
& \times\left(H_{2}(T)-H_{2}(0)\right) .
\end{aligned}
$$


c) $G_{1}(t, s) \geq \gamma_{1}(t) J_{1}(s), \forall(t, s) \in[0, T] \times[0, T]$, where

$$
\begin{aligned}
& \gamma_{1}(t)=\frac{1}{\tau_{1}}\left\{k_{1}(t)+\frac{1}{\Delta_{1}}\left[\left(T^{n-1}-t^{n-1}\right)\left(1-H_{2}(T)+H_{2}(0)\right)\right.\right. \\
& \left.+\int_{0}^{T}\left(T^{n-1}-\tau^{n-1}\right) d H_{2}(\tau)\right] \int_{0}^{T} k_{1}(\tau) d H_{1}(\tau) \\
& \left.+\frac{1}{\Delta_{1}}\left[t^{n-1}\left(1-H_{1}(T)+H_{1}(0)\right)+\int_{0}^{T} \tau^{n-1} d H_{1}(\tau)\right] \int_{0}^{T} k_{1}(\tau) d H_{2}(\tau)\right\} .
\end{aligned}
$$

Lemma 2.4. ([16]) Assume that $H_{1}, H_{2}:[0, T] \rightarrow \mathbb{R}$ are nondecreasing functions, $H_{1}(T)-H_{1}(0)<$ $1, H_{2}(T)-H_{2}(0)<1$, and $z \in C[0, T]$ with $z(t) \geq 0$ for all $t \in[0, T]$. Then the solution $u$ of problem (2.1)-(2.2), given in Lemma 2.1, satisfies the inequalities $u(t) \geq 0$ and $u(t) \geq \gamma_{1}(t) \max _{t^{\prime} \in[0, T]} u\left(t^{\prime}\right)$ for all $t \in[0, T]$.

We can also formulate similar results as Lemmas 2.1-2.4 for the ordinary differential equation

$$
v^{(m)}(t)+\widetilde{z}(t)=0, \quad 0<t<T,
$$

with the integral boundary conditions

$$
v(0)=\int_{0}^{T} v(s) d K_{1}(s), v^{\prime}(0)=\cdots=v^{(m-2)}(0)=0, v(T)=\int_{0}^{T} v(s) d K_{2}(s),
$$

where $m \in \mathbb{N}, m \geq 2, K_{1}, K_{2}:[0, T] \rightarrow \mathbb{R}$ are nondecreasing functions and $\widetilde{z} \in C[0, T]$. In the case $m=2$, the boundary conditions have the form $v(0)=\int_{0}^{T} v(s) d K_{1}(s), v(T)=\int_{0}^{T} v(s) d K_{2}(s)$. We denote by $\Delta_{2}, g_{2}, G_{2}, h_{2}, k_{2}, \tau_{2}, J_{2}$ and $\gamma_{2}$ the corresponding constants and functions for problem (2.5)-(2.6) defined in a similar manner as $\Delta_{1}, g_{1}, G_{1}, h_{1}, k_{1}, \tau_{1}, J_{1}$ and $\gamma_{1}$, respectively.

In the proof of our existence result, we shall use the Schauder fixed point theorem which we present now.

Theorem 2.5. Let $X$ be a Banach space and $Y \subset X$ a nonempty, bounded, convex and closed subset. If the operator $A: Y \rightarrow Y$ is completely continuous, then $A$ has at least one fixed point.

\section{Main Results}

We present the assumptions that we shall use in the sequel.

$(J 1) H_{1}, H_{2}, K_{1}, K_{2}:[0, T] \rightarrow \mathbb{R}$ are nondecreasing functions, $H_{1}(T)-H_{1}(0)<1, H_{2}(T)-$ $H_{2}(0)<1, K_{1}(T)-K_{1}(0)<1$ and $K_{2}(T)-K_{2}(0)<1$.

$(J 2)$ The functions $a, b:[0, T] \rightarrow[0, \infty)$ are continuous and there exist $t_{1}, t_{2} \in(0, T)$ such that $a\left(t_{1}\right)>0, b\left(t_{2}\right)>0$.

(J3) $f, g:[0, \infty) \rightarrow[0, \infty)$ are continuous functions and there exists $c_{0}>0$ such that $f(u)<\frac{c_{0}}{L}$, $g(u)<\frac{c_{0}}{L}$ for all $u \in\left[0, c_{0}\right]$, where $L=\max \left\{\int_{0}^{T} a(s) J_{1}(s) d s, \int_{0}^{T} b(s) J_{2}(s) d s\right\}$ and $J_{1}, J_{2}$ are defined in Section 2.

$(J 4) f, g:[0, \infty) \rightarrow[0, \infty)$ are continuous functions and satisfy the conditions $\lim _{u \rightarrow \infty} \frac{f(u)}{u}=\infty, \lim _{u \rightarrow \infty} \frac{g(u)}{u}=\infty$.

Our first theorem is the following existence result for problem $(S)-(B C)$.

Theorem 3.1. Assume that assumptions $(J 1)-(J 3)$ hold. Then problem $(S)-(B C)$ has at least one positive solution for $a_{0}>0$ and $b_{0}>0$ sufficiently small. 
Proof. By $(J 1)-(J 2)$, we deduce that $\int_{0}^{T} a(s) J_{1}(s) d s>0$ and $\int_{0}^{T} b(s) J_{2}(s) d s>0$, that is, the constant $L$ from $(J 3)$ is positive. We consider the problems

$$
\begin{aligned}
& \left\{h^{(n)}(t)=0, \quad t \in(0, T),\right. \\
& \left\{\begin{array}{l}
h(0)=\int_{0}^{T} h(s) d H_{1}(s)+1, h^{\prime}(0)=\cdots=h^{(n-2)}(0)=0, h(T)=\int_{0}^{T} h(s) d H_{2}(s),
\end{array}\right. \\
& \left\{\begin{array}{l}
k^{(m)}(t)=0, \quad t \in(0, T), \\
k(0)=\int_{0}^{T}, k(s) d K_{1}(s)+1, \quad k^{\prime}(0)=\cdots=k^{(m-2)}(0)=0, k(T)=\int_{0}^{T} k(s) d K_{2}(s) .
\end{array}\right.
\end{aligned}
$$

The above problems (3.1) and (3.2) have the solutions

$$
\begin{aligned}
& h(t)=\frac{1}{\Delta_{1}}\left[-t^{n-1}\left(1-\int_{0}^{T} d H_{2}(s)\right)+T^{n-1}-\int_{0}^{T} s^{n-1} d H_{2}(s)\right], t \in[0, T], \\
& k(t)=\frac{1}{\Delta_{2}}\left[-t^{m-1}\left(1-\int_{0}^{T} d K_{2}(s)\right)+T^{m-1}-\int_{0}^{T} s^{m-1} d K_{2}(s)\right], \quad t \in[0, T],
\end{aligned}
$$

respectively, where $\Delta_{1}$ and $\Delta_{2}$ are defined in Section 2. By assumption $(J 1)$ we obtain $h(t)>0$ and $k(t)>0$ for all $t \in[0, T)$.

We define the functions $x(t)$ and $y(t), t \in[0, T]$ by $x(t)=u(t)-a_{0} h(t)$ and $y(t)=v(t)-b_{0} k(t)$ for all $t \in[0, T]$, where $(u, v)$ is a solution of $(S)-(B C)$. Then $(S)-(B C)$ can be equivalently written as

$$
\left\{\begin{array}{l}
x^{(n)}(t)+a(t) f\left(y(t)+b_{0} k(t)\right)=0, \quad t \in(0, T), \\
y^{(m)}(t)+b(t) g\left(x(t)+a_{0} h(t)\right)=0, \quad t \in(0, T),
\end{array}\right.
$$

with the boundary conditions

$$
\left\{\begin{array}{l}
x(0)=\int_{0}^{T} x(s) d H_{1}(s), x^{\prime}(0)=\cdots=x^{(n-2)}(0)=0, \quad x(T)=\int_{0}^{T} x(s) d H_{2}(s), \\
y(0)=\int_{0}^{T} y(s) d K_{1}(s), y^{\prime}(0)=\cdots=y^{(m-2)}(0)=0, \quad y(T)=\int_{0}^{T} y(s) d K_{2}(s) .
\end{array}\right.
$$

Using the Green's functions $G_{1}$ and $G_{2}$ from Section 2, we find a pair $(x, y)$ is a solution of problem (3.4)-(3.5) if and only if $(x, y)$ is a solution for the nonlinear integral equations

$$
\left\{\begin{array}{l}
x(t)=\int_{0}^{T} G_{1}(t, s) a(s) f\left(\int_{0}^{T} G_{2}(s, \tau) b(\tau) g\left(x(\tau)+a_{0} h(\tau)\right) d \tau+b_{0} k(s)\right) d s, \\
y(t)=\int_{0}^{T} G_{2}(t, s) b(s) g\left(x(s)+a_{0} h(s)\right) d s, \quad 0 \leq t \leq T
\end{array}\right.
$$

where $h(t), k(t), t \in[0, T]$ are given by (3.3).

We consider the Banach space $X=C[0, T]$ with the supremum norm $\|\cdot\|$ and define the set $E=\left\{x \in C[0, T], 0 \leq x(t) \leq c_{0}, \forall t \in[0, T]\right\} \subset X$.

We also define the operator $\mathcal{A}: E \rightarrow X$ by

$$
\begin{gathered}
(\mathcal{A} x)(t)=\int_{0}^{T} G_{1}(t, s) a(s) f\left(\int_{0}^{T} G_{2}(s, \tau) b(\tau) g\left(x(\tau)+a_{0} h(\tau)\right) d \tau+b_{0} k(s)\right) d s, \\
0 \leq t \leq T, \quad x \in E .
\end{gathered}
$$

For sufficiently small $a_{0}>0$ and $b_{0}>0$, by $(J 3)$, we deduce that $f\left(y(t)+b_{0} k(t)\right) \leq \frac{c_{0}}{L}$ and $g\left(x(t)+a_{0} h(t)\right) \leq \frac{c_{0}}{L}$ for all $t \in[0, T]$ and $x, y \in E$. Then, by using Lemma 2.4, we obtain $(\mathcal{A} x)(t) \geq 0$ for all $t \in[0, T]$ and $x \in E$. 
By Lemma 2.3, for all $x \in E$, we have

$$
\begin{gathered}
\int_{0}^{T} G_{2}(s, \tau) b(\tau) g\left(x(\tau)+a_{0} h(\tau)\right) d \tau \leq \int_{0}^{T} J_{2}(\tau) b(\tau) g\left(x(\tau)+a_{0} h(\tau)\right) d \tau \\
\leq \frac{c_{0}}{L} \int_{0}^{T} J_{2}(\tau) b(\tau) d \tau \leq c_{0}, \quad \forall s \in[0, T]
\end{gathered}
$$

and

$$
\begin{gathered}
(\mathcal{A} x)(t) \leq \int_{0}^{T} J_{1}(s) a(s) f\left(\int_{0}^{T} G_{2}(s, \tau) b(\tau) g\left(x(\tau)+a_{0} h(\tau)\right) d \tau+b_{0} k(s)\right) d s \\
\leq \frac{c_{0}}{L} \int_{0}^{T} J_{1}(s) a(s) d s \leq c_{0}, \quad \forall t \in[0, T] .
\end{gathered}
$$

Therefore $\mathcal{A}(E) \subset E$.

Using standard arguments, we deduce that $\mathcal{A}$ is completely continuous. By Theorem 2.5, we conclude that $\mathcal{A}$ has a fixed point $x \in E$. This element together with $y$ given by (3.6) represents a solution for (3.4)-(3.5). This shows that our problem $(S)-(B C)$ has a positive solution $(u, v)$ with $u=x+a_{0} h, v=y+b_{0} k$ for sufficiently small $a_{0}$ and $b_{0}$.

In what follows, we present sufficient conditions for the nonexistence of positive solutions of $(S)-$ $(B C)$.

Theorem 3.2. Assume that assumptions $(J 1),(J 2)$ and $(J 4)$ hold. Then problem $(S)-(B C)$ has no positive solution for $a_{0}$ and $b_{0}$ sufficiently large.

Proof. We suppose that $(u, v)$ is a positive solution of $(S)-(B C)$. Then $(x, y)$ with $x=u-a_{0} h$, $y=v-b_{0} k$ is a solution for (3.4)-(3.5), where $h$ and $k$ are the solutions of problems (3.1) and (3.2), respectively, (given by (3.3)). By $(J 2)$ there exists $c \in(0, T / 2)$ such that $t_{1}, t_{2} \in(c, T-c)$, and then $\int_{c}^{T-c} a(s) J_{1}(s) d s>0, \int_{c}^{T-c} b(s) J_{2}(s) d s>0$. Now by using Lemma 2.4 , we have $x(t) \geq 0$, $y(t) \geq 0$ for all $t \in[0, T]$, and $\inf _{t \in[c, T-c]} x(t) \geq \gamma_{1}^{0}\|x\|$ and $\inf _{t \in[c, T-c]} y(t) \geq \gamma_{2}^{0}\|y\|$, where $\gamma_{1}^{0}=\inf _{t \in[c, T-c]} \gamma_{1}(t), \gamma_{2}^{0}=\inf _{t \in[c, T-c]} \gamma_{2}(t)$.

Using now (3.3), we deduce that

$$
\inf _{t \in[c, T-c]} h(t)=h(T-c)=\frac{h(T-c)}{h(0)}\|h\|, \inf _{t \in[c, T-c]} k(t)=k(T-c)=\frac{k(T-c)}{k(0)}\|k\| .
$$

Therefore, we obtain

$$
\begin{aligned}
& \inf _{t \in[c, T-c]}\left(x(t)+a_{0} h(t)\right) \geq \gamma_{1}^{0}\|x\|+a_{0} \frac{h(T-c)}{h(0)}\|h\| \geq r_{1}\left(\|x\|+a_{0}\|h\|\right) \geq r_{1}\left\|x+a_{0} h\right\|, \\
& \inf _{t \in[c, T-c]}\left(y(t)+b_{0} k(t)\right) \geq \gamma_{2}^{0}\|y\|+b_{0} \frac{k(T-c)}{k(0)}\|k\| \geq r_{2}\left(\|y\|+b_{0}\|k\|\right) \geq r_{2}\left\|y+b_{0} k\right\|,
\end{aligned}
$$

where $r_{1}=\min \left\{\gamma_{1}^{0}, \frac{h(T-c)}{h(0)}\right\}, r_{2}=\min \left\{\gamma_{2}^{0}, \frac{k(T-c)}{k(0)}\right\}$.

We now consider $R=\left(\min \left\{\gamma_{2}^{0} r_{1} \int_{c}^{T-c} b(s) J_{2}(s) d s, \gamma_{1}^{0} r_{2} \int_{c}^{T-c} a(s) J_{1}(s) d s\right\}\right)^{-1}>0$.

By using (J4), for $R$ defined above, we conclude that there exists $M>0$ such that $f(u)>$ $2 R u, g(u)>2 R u$ for all $u \geq M$. We consider $a_{0}>0$ and $b_{0}>0$ sufficiently large such that $\inf _{t \in[c, T-c]}\left(x(t)+a_{0} h(t)\right) \geq M$ and $\inf _{t \in[c, T-c]}\left(y(t)+b_{0} k(t)\right) \geq M$. By (J2), (3.4), (3.5) and the above inequalities, we deduce that $\|x\|>0$ and $\|y\|>0$. 
Now by using Lemma 2.3 and the above considerations, we have

$$
\begin{aligned}
& y(c)=\int_{0}^{T} G_{2}(c, s) b(s) g\left(x(s)+a_{0} h(s)\right) d s \geq \int_{0}^{T} \gamma_{2}(c) J_{2}(s) b(s) g\left(x(s)+a_{0} h(s)\right) d s \\
& \geq \gamma_{2}^{0} \int_{c}^{T-c} J_{2}(s) b(s) g\left(x(s)+a_{0} h(s)\right) d s \geq 2 R \gamma_{2}^{0} \int_{c}^{T-c} J_{2}(s) b(s)\left(x(s)+a_{0} h(s)\right) d s \\
& \geq 2 R \gamma_{2}^{0} \int_{c}^{T-c} J_{2}(s) b(s) \inf _{\tau \in[c, T-c]}\left(x(\tau)+a_{0} h(\tau)\right) d s \\
& \geq 2 R \gamma_{2}^{0} r_{1} \int_{c}^{T-c} J_{2}(s) b(s)\left\|x+a_{0} h\right\| d s \geq 2\left\|x+a_{0} h\right\| \geq 2\|x\| .
\end{aligned}
$$

Therefore, we obtain

$$
\|x\| \leq y(c) / 2 \leq\|y\| / 2
$$

In a similar manner, we deduce

$$
\begin{aligned}
& x(c)=\int_{0}^{T} G_{1}(c, s) a(s) f\left(y(s)+b_{0} k(s)\right) d s \geq \int_{0}^{T} \gamma_{1}(c) J_{1}(s) a(s) f\left(y(s)+b_{0} k(s)\right) d s \\
& \geq \gamma_{1}^{0} \int_{c}^{T-c} J_{1}(s) a(s) f\left(y(s)+b_{0} k(s)\right) d s \geq 2 R \gamma_{1}^{0} \int_{c}^{T-c} J_{1}(s) a(s)\left(y(s)+b_{0} k(s)\right) d s \\
& \geq 2 R \gamma_{1}^{0} \int_{c}^{T-c} J_{1}(s) a(s) \inf _{\tau \in[c, T-c]}\left(y(\tau)+b_{0} k(\tau)\right) d s \\
& \geq 2 R \gamma_{1}^{0} r_{2} \int_{c}^{T-c} J_{1}(s) a(s)\left\|y+b_{0} k\right\| d s \geq 2\left\|y+b_{0} k\right\| \geq 2\|y\| .
\end{aligned}
$$

So, we obtain

$$
\|y\| \leq x(c) / 2 \leq\|x\| / 2
$$

By (3.7) and (3.8), we conclude that $\|x\| \leq\|y\| / 2 \leq\|x\| / 4$, which is a contradiction, because $\|x\|>0$. Then, for $a_{0}$ and $b_{0}$ sufficiently large, our problem $(S)-(B C)$ has no positive solution.

Similar results as Theorems 3.1 and 3.2 can be obtained if instead of boundary conditions $(B C)$ we have

$\left(B C_{1}\right)\left\{\begin{array}{l}u(0)=\int_{0}^{T} u(s) d H_{1}(s), u^{\prime}(0)=\cdots=u^{(n-2)}(0)=0, u(T)=\int_{0}^{T} u(s) d H_{2}(s)+a_{0}, \\ v(0)=\int_{0}^{T} v(s) d K_{1}(s), v^{\prime}(0)=\cdots=v^{(m-2)}(0)=0, \quad v(T)=\int_{0}^{T} v(s) d K_{2}(s)+b_{0},\end{array}\right.$

or

$\left(B C_{2}\right)\left\{\begin{array}{l}u(0)=\int_{0}^{T} u(s) d H_{1}(s)+a_{0}, u^{\prime}(0)=\cdots=u^{(n-2)}(0)=0, u(T)=\int_{0}^{T} u(s) d H_{2}(s), \\ v(0)=\int_{0}^{T} v(s) d K_{1}(s), v^{\prime}(0)=\cdots=v^{(m-2)}(0)=0, v(T)=\int_{0}^{T} v(s) d K_{2}(s)+b_{0},\end{array}\right.$

or

$\left(B C_{3}\right)\left\{\begin{array}{l}u(0)=\int_{0}^{T} u(s) d H_{1}(s), u^{\prime}(0)=\cdots=u^{(n-2)}(0)=0, u(T)=\int_{0}^{T} u(s) d H_{2}(s)+a_{0}, \\ v(0)=\int_{0}^{T} v(s) d K_{1}(s)+b_{0}, v^{\prime}(0)=\cdots=v^{(m-2)}(0)=0, \quad v(T)=\int_{0}^{T} v(s) d K_{2}(s),\end{array}\right.$

where $a_{0}$ and $b_{0}$ are positive constants. 
For problem $(S)-\left(B C_{1}\right)$, instead of functions $h$ and $k$ from the proof of Theorem 3.1, the solutions of problems

$$
\begin{aligned}
& \left\{\begin{array}{l}
\widetilde{h}^{(n)}(t)=0, t \in(0, T), \\
\widetilde{h}(0)=\int_{0}^{T} \widetilde{h}(s) d H_{1}(s), \widetilde{h}^{\prime}(0)=\cdots=\widetilde{h}^{(n-2)}(0)=0, \widetilde{h}(T)=\int_{0}^{T} \widetilde{h}(s) d H_{2}(s)+1,
\end{array}\right. \\
& \left\{\begin{array}{l}
\widetilde{k}^{(m)}(t)=0, \quad t \in(0, T), \\
\widetilde{k}(0)=\int_{0}^{T} \widetilde{k}(s) d K_{1}(s), \quad \widetilde{k}^{\prime}(0)=\cdots=\widetilde{k}^{(m-2)}(0)=0, \widetilde{k}(T)=\int_{0}^{T} \widetilde{k}(s) d K_{2}(s)+1
\end{array}\right.
\end{aligned}
$$

are

$$
\begin{aligned}
& \widetilde{h}(t)=\frac{1}{\Delta_{1}}\left[t^{n-1}\left(1-\int_{0}^{T} d H_{1}(s)\right)+\int_{0}^{T} s^{n-1} d H_{1}(s)\right], t \in[0, T], \\
& \widetilde{k}(t)=\frac{1}{\Delta_{2}}\left[t^{m-1}\left(1-\int_{0}^{T} d K_{1}(s)\right)+\int_{0}^{T} s^{m-1} d K_{1}(s)\right], t \in[0, T],
\end{aligned}
$$

respectively. By assumption $(J 1)$ we obtain $\widetilde{h}(t)>0$ and $\widetilde{k}(t)>0$ for all $t \in(0, T]$.

For problem $(S)-\left(B C_{2}\right)$, instead of functions $h$ and $k$ from the proof of Theorem 3.1, the solutions of problems (3.1) and (3.10) are the functions $h$ and $\widetilde{k}$, respectively, which satisfy $h(t)>0$ for all $t \in[0, T)$ and $\widetilde{k}(t)>0$ for all $t \in(0, T]$. For problem $(S)-\left(B C_{3}\right)$, instead of functions $h$ and $k$ from the proof of Theorem 3.1, the solutions of problems (3.9) and (3.2) are the functions $\widetilde{h}$ and $k$, respectively, which satisfy $\widetilde{h}(t)>0$ for all $t \in(0, T]$ and $k(t)>0$ for all $t \in[0, T)$.

Therefore we also obtain the following results.

Theorem 3.3. Assume that assumptions $(J 1)-(J 3)$ hold. Then problem $(S)-\left(B C_{1}\right)$ has at least one positive solution $(u(t)>0$ and $v(t)>0$ for all $t \in(0, T])$ for $a_{0}>0$ and $b_{0}>0$ sufficiently small.

Theorem 3.4. Assume that assumptions $(J 1)$, $(J 2)$ and $(J 4)$ hold. Then problem $(S)-\left(B C_{1}\right)$ has no positive solution $(u(t)>0$ and $v(t)>0$ for all $t \in(0, T])$ for $a_{0}$ and $b_{0}$ sufficiently large.

Theorem 3.5. Assume that assumptions $(J 1)-(J 3)$ hold. Then problem $(S)-\left(B C_{2}\right)$ has at least one positive solution $(u(t)>0$ for all $t \in[0, T)$, and $v(t)>0$ for all $t \in(0, T])$ for $a_{0}>0$ and $b_{0}>0$ sufficiently small.

Theorem 3.6. Assume that assumptions $(J 1),(J 2)$ and $(J 4)$ hold. Then problem $(S)-\left(B C_{2}\right)$ has no positive solution $(u(t)>0$ for all $t \in[0, T)$, and $v(t)>0$ for all $t \in(0, T])$ for $a_{0}$ and $b_{0}$ sufficiently large.

Theorem 3.7. Assume that assumptions $(J 1)-(J 3)$ hold. Then problem $(S)-\left(B C_{3}\right)$ has at least one positive solution $(u(t)>0$ for all $t \in(0, T]$, and $v(t)>0$ for all $t \in[0, T))$ for $a_{0}>0$ and $b_{0}>0$ sufficiently small.

Theorem 3.8. Assume that assumptions $(J 1),(J 2)$ and $(J 4)$ hold. Then problem $(S)-\left(B C_{3}\right)$ has no positive solution $(u(t)>0$ for all $t \in(0, T]$, and $v(t)>0$ for all $t \in[0, T))$ for $a_{0}$ and $b_{0}$ sufficiently large. 


\section{An Example}

We consider $T=1, n=3, m=4, a(t)=a t^{2}, b(t)=b t^{3}$, for all $t \in[0,1]$ with $a, b>0, H_{1}(t)=\frac{t^{4}}{3}$, $K_{2}(t)=t^{3} / 2$, and

$$
H_{2}(t)=\left\{\begin{array}{ll}
0, & t \in[0,1 / 3), \\
1 / 3, & t \in[1 / 3,2 / 3), \\
5 / 6, & t \in[2 / 3,1],
\end{array} \quad K_{1}(t)= \begin{cases}0, & t \in[0,1 / 2), \\
1 / 2, & t \in[1 / 2,1] .\end{cases}\right.
$$

Then, we have $\int_{0}^{1} u(s) d H_{1}(s)=\frac{4}{3} \int_{0}^{1} s^{3} u(s) d s, \int_{0}^{1} u(s) d H_{2}(s)=\frac{1}{3} u\left(\frac{1}{3}\right)+\frac{1}{2} u\left(\frac{2}{3}\right), \int_{0}^{1} v(s) d K_{1}(s)=$ $\frac{1}{2} v\left(\frac{1}{2}\right), \int_{0}^{1} v(s) d K_{2}(s)=\frac{3}{2} \int_{0}^{1} s^{2} v(s) d s$. We also consider the functions $f, g:[0, \infty) \rightarrow[0, \infty)$, $f(x)=\frac{\widetilde{a} x^{\alpha}}{x^{\beta}+\widetilde{c}}, g(x)=\frac{\widetilde{b} x^{\gamma}}{x^{\delta}+d}$ for all $x \in[0, \infty)$, with $\widetilde{a}, \widetilde{b}, \widetilde{c}, \widetilde{d}>0, \alpha, \beta, \gamma, \delta>0, \alpha>\beta+1$, and $\gamma>\delta+1$. We have $\lim _{x \rightarrow \infty} f(x) / x=\lim _{x \rightarrow \infty} g(x) / x=\infty$.

Therefore, we consider the nonlinear higher-order differential system

$$
\left\{\begin{array}{l}
u^{(3)}(t)+a t^{2} \frac{\widetilde{a} v^{\alpha}(t)}{v^{\beta}(t)+\widetilde{c}}=0, \quad t \in(0,1), \\
v^{(4)}(t)+b t^{3} \frac{\widetilde{b} u^{\gamma}(t)}{u^{\delta}(t)+\widetilde{d}}=0, \quad t \in(0,1)
\end{array}\right.
$$

with the boundary conditions

$$
\left\{\begin{array}{l}
u(0)=\frac{4}{3} \int_{0}^{1} s^{3} u(s) d s+a_{0}, \quad u^{\prime}(0)=0, \quad u(1)=\frac{1}{3} u\left(\frac{1}{3}\right)+\frac{1}{2} u\left(\frac{2}{3}\right) \\
v(0)=\frac{1}{2} v\left(\frac{1}{2}\right)+b_{0}, \quad v^{\prime}(0)=v^{\prime \prime}(0)=0, \quad v(1)=\frac{3}{2} \int_{0}^{1} s^{2} v(s) d s
\end{array}\right.
$$

Then, we obtain $H_{1}(1)-H_{1}(0)=\frac{1}{3}<1, H_{2}(1)-H_{2}(0)=\frac{5}{6}<1, K_{1}(1)-K_{1}(0)=\frac{1}{2}<1$ and $K_{2}(1)-K_{2}(0)=\frac{1}{2}<1$. We deduce that assumptions $(J 1),(J 2)$ and $(J 4)$ are satisfied. We also obtain $\Delta_{1}=\frac{43}{81}, \Delta_{2}=\frac{13}{32}, \tau_{1}=\frac{123}{43}, \tau_{2}=\frac{34}{13}, h_{1}(s)=s(1-s)^{2}, h_{2}(s)=\frac{1}{2} s(1-s)^{3}$, $J_{1}(s)=\frac{123}{43} s(1-s)^{2}$, and $J_{2}(s)=\frac{17}{13} s(1-s)^{3}, s \in[0,1]$.

By using the above functions $J_{1}$ and $J_{2}$, we deduce $\widetilde{A}=\int_{0}^{1} s^{2} J_{1}(s) d s \approx 0.04767442, \widetilde{B}=$ $\int_{0}^{1} s^{3} J_{2}(s) d s \approx 0.00467033$, and then $L=\max \{a \widetilde{A}, b \widetilde{B}\}$. We choose $c_{0}=1$ and if we select $\widetilde{a}, \widetilde{b}, \widetilde{c}, \tilde{d}$ satisfying the conditions $\widetilde{a}<\frac{1+\widetilde{c}}{L}=(1+\widetilde{c}) \min \left\{\frac{1}{a \widetilde{A}}, \frac{1}{b \widetilde{B}}\right\}, \widetilde{b}<\frac{1+\widetilde{d}}{L}=(1+\widetilde{d}) \min \left\{\frac{1}{a \widetilde{A}}, \frac{1}{b \widetilde{B}}\right\}$, then we conclude that $f(x) \leq \frac{\widetilde{a}}{1+\widetilde{c}}<\frac{1}{L}, g(x) \leq \frac{\widetilde{b}}{1+\widetilde{d}}<\frac{1}{L}$ for all $x \in[0,1]$. For example, if $a=2, b=3$, $\widetilde{c}=\widetilde{d}=1$, then for $\widetilde{a} \leq 20.97$ and $\widetilde{b} \leq 20.97$ the above conditions for $f$ and $g$ are satisfied. So, assumption (J3) is also satisfied. By Theorems 3.1 and 3.2 we deduce that problem $\left(S_{0}\right)-\left(B C_{0}\right)$ has at least one positive solution (here $u(t)>0$ and $v(t)>0$ for all $t \in[0,1]$ ) for sufficiently small $a_{0}>0$ and $b_{0}>0$, and no positive solution for sufficiently large $a_{0}$ and $b_{0}$.

\section{Conclusion}

In this paper, we studied the system of nonlinear higher-order ordinary differential equations $(S)$ with the Riemann-Stieltjes integral boundary conditions $(B C)$ which contain some positive constants. By using the Schauder fixed point theorem and some properties of the associated Green's functions, we show that this problem has at least one positive solution for sufficiently small constants. Then, we give sufficient conditions for the nonexistence of positive solutions. Similar results for other three boundary value problems are also presented.

\section{Competing Interests}

Authors have declared that no competing interests exist. 


\section{References}

[1] Henderson J, Luca R. On a system of higher-order multi-point boundary value problems. Electronic Journal of Qualitative Theory of Differential Equations. 2012;2012:49:1-14.

[2] Cui Y, Sun J. On existence of positive solutions of coupled integral boundary value problems for a nonlinear singular superlinear differential system. Electronic Journal of Qualitative Theory of Differential Equations. 2012;2012:41:1-13.

[3] Goodrich CS. Nonlocal systems of BVPs with asymptotically superlinear boundary conditions. Commentationes Mathematicae Universitatis Carolinae. 2012;53:79-97.

[4] Henderson J, Luca R. Boundary Value Problems for Systems of Differential, Difference and Fractional Equations. Positive Solutions. Elsevier. Amsterdam; 2016.

[5] Henderson J, Luca R, Tudorache A. On a system of fractional differential equations with coupled integral boundary conditions. Fractional Calculus and Applied Analysis. 2015;8(2):361-386.

[6] Henderson J, Ntouyas SK. Positive solutions for systems of nonlinear boundary value problems. Nonlinear Studies. 2008;15:51-60.

[7] Infante G, Minhos FM, Pietramala P. Non-negative solutions of systems of ODEs with coupled boundary conditions. Communications in Nonlinear Science and Numerical Simulation. 2012;17:4952-4960.

[8] Ji Y, Guo Y. The existence of countably many positive solutions for some nonlinear $n$th order $m$-point boundary value problems. Journal of Computational and Applied Mathematics. 2009;232:187-200.

[9] Lan KQ. Positive solutions of systems of Hammerstein integral equations. Communications in Applied Analysis. 2011;15:521-528.

[10] Luca R. Existence of positive solutions for a class of higher-order $m$-point boundary value problems. Electronic Journal of Qualitative Theory of Differential Equations. 2010;2010:74:115.

[11] Luca R. Positive solutions for a higher-order $m$-point boundary value problem. Mediterranean Journal of Mathematics. 2012;9:381-394.

[12] Ma R, Thompson B. Positive solutions for nonlinear $m$-point eigenvalue problems. Journal of Mathematical Analysis and Applications. 2004;297:24-37.

[13] Song W, Gao W. Positive solutions for a second-order system with integral boundary conditions. Electronic Journal of Differential Equations. 2011;2011:13:1-9.

[14] Su H, Wei Z, Zhang X, Liu J. Positive solutions of $n$-order and $m$-order multi-point singular boundary value system. Applied Mathematics and Computation. 2007;188:1234-1243.

[15] Yang Z, Zhang Z. Positive solutions for a system of nonlinear singular Hammerstein integral equations via nonnegative matrices and applications. Positivity. 2012;16:783-800.

[16] Luca R, Tudorache A. Existence of positive solutions to a system of higher-order semipositone integral boundary value problems. Communications in Applied Analysis. 2015;19:589-604.

(C)2016 Luca and Tudorache; This is an Open Access article distributed under the terms of the Creative Commons Attribution License (http://creativecommons.org/licenses/by/4.0), which permits unrestricted use, distribution, and reproduction in any medium, provided the original work is properly cited.

Peer-review history:

The peer review history for this paper can be accessed here (Please copy paste the total link in your browser address bar)

http://sciencedomain.org/review-history/16681 